\title{
The Semantic Theory of Truth: Field's Incompleteness Objection
}

\section{Glen A. Hoffmann}

Ryerson University

digital.library.ryerson.ca/object/219

Please Cite:

Hoffmann, G. A. (2007). The semantic theory of truth: Field's incompleteness objection. Philosophia 35(2), 161-170.

$\underline{\text { doi:10.1007/s11406-007-9057-9 }}$

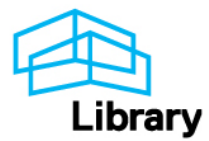




\title{
The Semantic Theory of Truth: Field's Incompleteness Objection
}

\author{
Glen Hoffmann \\ Ryerson University
}

\section{\$1 Introduction}

The semantic theory of truth is outlined and defended by Tarski in 'The Concept of Truth in Formalized Languages' (1931) and 'The Semantic Conception of Truth and the Foundations of Semantics' (1944). ${ }^{1}$ The heart of this theory is Tarski's semantic definition of truth wherein truth is explicitly defined, for various formalized languages $\mathrm{L}_{1}-\mathrm{L}_{\mathrm{n}}$ in terms of three semantic concepts: satisfaction, denotation, and application. In general terms, Tarski defines truth for any sentence s of $\mathrm{L}$ in terms of the denotation conditions of all names $\mathrm{N}$, the application conditions of all predicates $\mathrm{P}$, and the satisfaction conditions of all functions symbols $\mathrm{F}$ contained in $\mathrm{s}$. As is well known, Tarski's definition is constructed compositionally, i.e., in such a way that the semantic properties of complex expressions are recursively built up from the semantic properties of their primitive components via uncontroversial logical machinery. Crucial features of the definition include the fact that it satisfies the formal criteria of material adequacy, ${ }^{2}$ noncircularity, and freedom from paradox. ${ }^{3}$

\footnotetext{
${ }^{1}$ Both reprinted in Tarski's Logic, Semantics, Metamathematics (1983). References to the former are from the reprint, to the latter are from the original.

${ }^{2}$ This means that it implies, for each declarative sentence of the language, a sentence of the form

(T) $\mathrm{s}$ is true-in- $\mathrm{L}$ if and only if $\mathrm{p}$,

where 's' has as substitution instances names of sentences in L and 'p' has as substitution instances translations of such object-language sentences. Insofar as it satisfies Convention T, then, the semantic definition of truth for the English language should yield as consequences the sentences
} 
In the seminal article 'Tarski's Theory of Truth' (1972), ${ }^{4}$ Field levels his venerable incompleteness objection against the semantic theory of truth. According to Field, the semantic theory of truth is unsatisfactory since the definition at its basis is incomplete in that it does not explain reference in primitive, physicalistic terms. While Field eventually abandoned the incompleteness objection in the course of his transition from correspondence theorist to deflationist, ${ }^{5}$ its considerable influence in the contemporary philosophical landscape is indisputable. Among other things, it has inspired a host of similar objections to the semantic theory of truth (e.g., Harman (1975), Benacerraf (1973), Pyne-Parsons (1973), Putnam (1981), Blackburn (1984), and Soames (1984)), and it continues to shape philosophical speculation into the definability and reducibility of the truth predicate (e.g., see Davidson (1996) and Wolenski (2001)).

In this paper, I defend the semantic theory of truth against Field's incompleteness objection. I first argue that a familiar reply to the incompleteness objection, endorsed by Davidson and McDowell (among others), is wrongheaded. I then argue that, within the constraints of a non-reductive physicalism and a local holism (one concerning the concepts of truth, reference and meaning), a more promising reply to Field's incompleteness objection can be fashioned.

(TI) 'snow is white' is true-in-English iff snow is white, (TI) 'grass is green' is true-in-English iff grass is green,

and correspondingly for each declarative sentence of the language. For more on the material adequacy condition, otherwise referred to as 'Convention T', see Tarski (1944), pp. 343-5.

${ }^{3}$ See Tarski (1944), pp. $343 \mathrm{ff}$ for more on how his definition satisfies such formal criteria.

${ }^{4}$ Later reprinted in Field (2001). All references are to the original. 


\section{\$2 The Incompleteness Objection}

According to the incompleteness objection (hereafter 'IC'), the definition at the basis of Tarski's theory fulfilled an important objective: it defined truth in terms of three core semantic concepts - satisfaction, denotation, and application (we can collectively label these concepts 'reference') — and the relations they bear to one another. ${ }^{6}$ The problem is that the semantic definition of truth is unsatisfactory since Tarski failed to properly define reference itself. Instead, it is claimed, Tarski merely furnished us with the extension of the reference predicate: the object-level axioms that govern the correct application of this predicate for a sizable class of formalized languages. For example, Tarski's definition of denotation for a natural language such as English would run as follows:

(D) To say that a name $\mathrm{N}$ denotes a given object $\mathrm{A}$ is the same as to stipulate that either $\mathrm{A}$ is France and $\mathrm{N}$ is 'France', or A is Germany and $\mathrm{N}$ is 'Germany', or...

where in the blanks go similar clauses, one for each name in the English language. His definition of application for the English language would run along similar lines:

(A) To say that a predicate $\mathrm{P}$ applies to a set of objects $\mathrm{A}$ is the same as to stipulate that either $\mathrm{A}$ is a state and $\mathrm{P}$ is statehood, $\mathrm{A}$ is tall and $\mathrm{P}$ is tallness, or...

\footnotetext{
${ }^{5}$ See, for example, the postscript to Field (1972) in his (2001).

${ }^{6}$ According to Field, this is no minor accomplishment. One application of such a definition, Field contends, is its relevance to higher-order logic, i.e., that it can be used to clarify important concepts in model theory. See his (1972), p. 351.
} 
where in the blanks go similar clauses, one for each predicate in the English language. ${ }^{7}$

In short, according to IC, the problem with the semantic definition of truth is that Tarski provides us with only part of a complete definition since he fails to supplement (D) and (A) with something more austere: a reduction of its base clauses. More specifically, there are two stringent desiderata Tarski's definition of truth fails to satisfy. 1) Tarski failed to reduce reference to non-semantic terms. 2) Tarski failed to reduce reference to physical terms. ${ }^{8}$ As Field puts the first of these points (1972, p.347):

Tarski succeeded in reducing the notion of truth to certain other semantic notions; but he did not in any way explicate these other notions, so that his results ought to make the word 'true' acceptable only to someone who already regarded these other semantic notions as acceptable.

On IC, then, the semantic definition of truth is incomplete from the perspective of physicalism, roughly, the perspective according to which every fact about the universe is ultimately explainable in terms of the vocabulary of physics and/or logico-mathematics. ${ }^{9}$ In this way, the semantic definition of truth is similar to a physicalistically non-reductive definition of the chemical concept of valence, ${ }^{10}$ one wherein 'valence' is defined by giving its extension, i.e., listing all the elements along with their valences along the following lines:

\footnotetext{
${ }^{7}$ Since Tarski defined satisfaction in terms of denotation and application, he did not require a freestanding definition of satisfaction.

${ }^{8}$ As Field notes, Tarski failed to reduce reference physicalistically in accordance with either the standards of so-called 'strict reduction' (whereby the bridging laws involve a high degree of precision) or 'approximate reduction' (whereby the bridging laws involve less precision). See his (1972), pp. 357ff. For our purposes, the distinction can be ignored.

${ }^{9}$ See Field's (1972), pp. 357-9, for a sensitive discussion of physicalism and its relation to (IC), although Field's formulation of this doctrine deviates slightly from my own. See also McDowell (1978), pp. 137ff concerning this issue.
} 
(V) $(\forall \mathrm{e})(\forall \mathrm{n})(\mathrm{e}$ has valence $\mathrm{n} \equiv \mathrm{e}$ is potassium and $\mathrm{n}$ is +1 , or e is sulphur and $\mathrm{n}$ is -2 , or ...)

where in the blanks goes a list of similar clauses, one for each element (Field (1972), p. 363). ${ }^{11}$ Such a definition of valence, similar to Tarski's definition of truth, is physicalistically incomplete since it does not supply us with a physicalistic reduction of its definiens: it fails to reduce 'valence' to the concepts of a natural science that is more fundamental than chemistry; for example, it does not, pace physics, explain the chemical properties of the elements in terms of the structural properties of the atoms out of which they are composed.

Most importantly, on IC, the physicalistic incompleteness of the semantic definition of truth seems to imply, ipso facto, that it is conceptually deficient: that it does not fully capture the content of the predicate 'is true' in the languages for which it is defined. Although Field is not explicit on this point, such an interpretation of IC seems unassailable insofar as it underwritten by a ceteris paribus straightforward inference: assuming physicalism, physicalistically incomplete definitions such as Tarski's definition of truth must, to some extent, be conceptually deficient, inadequate, or unilluminating. The inference from the physicalistic incompleteness to the conceptual deficiency thesis of Tarski’s definition seems to have a clear-cut, albeit contentious, rationale. ${ }^{12}$ Definitions of concepts (at least semantic concepts), the assumption seems to be, are subject to what might be called a 'conceptual adequacy constraint': they should employ definiens that are

\footnotetext{
${ }^{10}$ The valence of a chemical element is an integer associated with that element that represents the sorts of chemical combinations the element will enter into

${ }^{11}$ See Leeds (1978), pp. 120-3, for an illuminating discussion of this definition.

${ }^{12}$ In $\$ 4$ this rationale is called into question.
} 
simpler, more basic or more primitive than their definiendum. The central difficulty with the semantic definition of truth in this connection seems to be that it fails to satisfy any such constraint since Tarski defines the concept of truth in terms of the equally fundamental concept of reference without defining reference in more primitive terms (e.g., physical terms).

In the end, then, Field's argument seems to spring from the conjunction of physicalism and the conceptual adequacy constraint. The reasoning can be rendered precise: assuming pace physicalism that physical concepts, contra semantic concepts, are the most fundamental concepts and pace the conceptual adequacy constraint that definitions (at least of semantic concepts) must employ definiens that are more fundamental than their definiendum, Tarski's failure to define the semantic concept of reference in non-semantic terms renders it conceptually deficient; in particular, Tarski's failure to define the concept of reference in physical terms implies it cannot be fully conceptually illuminating.

\section{§3 Davidson and McDowell's Reply}

In defending Tarski's semantic theory of truth against IC a wide variety of replies are available to Field's opponent. I want to focus on two possible candidates here, the second of which appears to be superior. I discuss the first reply in this section and the second reply in the next section.

The first reply to Field's IC is that which has been proposed in broad outline by Davidson and McDowell (hereafter D\&M) (Davidson (1977, 1979) (McDowell 
(1978)). ${ }^{13}$ This reply involves rejecting the two central conclusions of IC. D\&M grant Field's premises that some form of physicalism is warranted, and that a physicalistically exemplary definition of truth should be physicalistically complete, but reject Field's conclusions that the semantic definition of truth is i) physicalistically incomplete (his stated conclusion) and ii) conceptually deficient (his tacit conclusion). Since Field's socalled 'fundamental desiderata' — that a definition of truth must define the semantic definiens in non-semantic and primitive, physicalistic terms - lack authority, D\&M claim, his physicalistic and conceptual deficiency conclusions can be dismissed out of hand.

The fundamental desiderata, and by implication the physicalistic and conceptual deficiency theses, lack authority, D\&M argue, since reference is not a concept that is susceptible of a reductive, physicalistic explanation of the sort Field envisages.

According to D\&M, reference is not physicalistically reducible since it is not a primitive semantic concept in respect of explanatory significance, i.e., since it "plays no essential role in explaining the relation between language and reality" (Davidson (1977), p.253). Instead, the argument runs, reference has a purely theoretical role: it is something like a hypothetical construct that we postulate in order to characterize other more fundamental semantic concepts and that we know everything about when we know how it operates to characterize them. This represents the core of D\&M's instrumentalist view of reference: the only purpose of the concept of reference is a theoretical one - to enable us to better

\footnotetext{
${ }^{13}$ Since these rejoinders were arrived at independently by Davidson and McDowell, they differ at least in point of detail. The focus here is on the common core of their arguments. See Wallace (1977) and Stalnaker (1984) for rejoinders to (IC) similar to Davidson's and McDowell's. See Field's (2001, chapter 3) for a brief discussion of Wallace's and Stalnaker's version of the incompleteness objection.
} 
characterize more primitive, lower-level concepts. ${ }^{14}$

The pivotal question is what grounds the instrumentalism/anti-realism about reference - that reference isn't an explanatory concept or an existent relation - at the basis of D\&M's reply to IC. Although the specific details are a matter of considerable disputation, D\&M's instrumentalism/anti-realism about reference seems to stem from some form or another of an anti-atomistic account of meaning bearing. According to antiatomism, sentences (or sub-sentential expressions), languages or theories are the primary vehicles of meaning rather than words, and if words have meaning at all it is derivative of their role in sentences, languages or theories. ${ }^{15}$ The reasoning is: assuming, viz. antiatomism, that words are not the primary vehicles of meaning, 'refers to' lacks primitive, theoretical significance, since 'refers to', by definition, is an atomistic relation - a relation between words on the one hand and whatever things those words are supposed to provide deliverances about on the other hand.

It would seem, though, that D\&M's rejoinder to IC misses its target. Where does it go wrong? Since it seems to me that some variety or another of anti-atomism (molecularist, holist or otherwise) is warranted, and since there is currently much agreement on this point, it would be imprudent to challenge the anti-atomistic basis of D\&M's reply to IC.

A better place to challenge D\&M's argument is in its later stages: even supposing some form of anti-atomism is warranted, D\&M's instrumentalism/anti-realism about

\footnotetext{
${ }^{14} \mathrm{An}$ anti-realism about reference seems to follow more or less as a direct consequence: reference is not an existent relation between expressions and whatever expressions are supposed to provide deliverances about. See Davidson's (1977), especially pp. 223-5, for more on the instrumentalist/anti-realist view of reference.

${ }^{15}$ I leave open the question of whether (D\&M) were advancing a form of molecularism (according to which sentences or sub-sentential expressions are the primary bearers of meaning) or holism (according to which whole languages or theories are the primary bearers of meaning) as the basis of their reply to (IC) since this question does not impinge on the critique of $(\mathrm{D} \& \mathrm{M})$ developed in the text.
} 
reference would not seem to follow. To appreciate this, notice that anti-atomism, on the above formulation, is committed to the thesis that sentences, sets of sentences, languages, or theories rather than words, are the primary vehicles of meaning (hereafter 'the priority thesis'). Formulated in this way, there is some flexibility in the interpretation of such a theory. Anti-atomism, in addition to its priority thesis, can be interpreted as being committed to one of two adjunct theses that divide its proponents into two broad categories:

$\left(\mathrm{AA}_{1}\right)$ The thesis that words are devoid of meaning,

or

$\left(\mathrm{AA}_{2}\right)$ The thesis that words (or at least some words) are meaningful but only in the derivative sense, as a function of their role in sentences, languages, or theories.

Keeping in mind that D\&M's argument against Field can be construed as an attempt to invoke $\left(\mathrm{AA}_{1}\right)$ or $\left(\mathrm{AA}_{2}\right)$ anti-atomism in support of an instrumentalism/antirealism account of reference, two features of this argument can be seen to engender its ultimate failure: 1) The grounds advanced by D\&M in support of anti-atomism establish $\left(\mathrm{AA}_{2}\right)$ anti-atomism at the upper limit, and 2) $\left(\mathrm{AA}_{2}\right)$ anti-atomism does not entail a (D\&M)-style instrumentalism/anti-realism about reference.

Consider 1), the grounds advanced by D\&M in support of anti-atomism. There is no mystery about the fact that D\&M, in the first instance, consider Quinean/Davidsonian arguments for the inscrutability of reference and meaning (Quine (1969) and Davidson (1979)) to establish anti-atomism: arguments to the effect that for any expression E and 
object $\mathrm{O}$, empirical evidence underdetermines whether $\mathrm{E}$ refers to or means $\mathrm{O}$. Now even granting the cogency of inscrutability arguments of this type, and there are many who don't, they do not establish the indeterminacy of reference/meaning: the fact that empirical evidence underdetermines referential and meaning ascriptions does not imply that word or sentence reference/meaning are indeterminate. Many, including Davidson himself (1979), concede the speciousness of this type of inference in full generality. In point of fact, the inference is often taken to be suspect since it relies on a divisive empiricist assumption, i.e., that the only evidence available for referential and meaning ascriptions is empirical. At a minimum, then, the invocation of inscrutability arguments to ground an indeterminacy thesis about reference and meaning is, we might say, a controversial maneuver undertaken by D\&M: one that can be called into question from a variety of anti-empiricist perspectives.

The implication is that $\mathrm{D} \& \mathrm{M}$ are far from establishing the indeterminacy of reference/meaning. At the upper limit, Quinean/Davidsonian inscrutability arguments show that word-meaning (or word-reference) might be derivative (as opposed to indeterminate), i.e., it might in some sense be a function of the role words play in sentences, languages, or theories. In short, absent acquiescence to substantive empiricist assumptions, D\&M's arguments for anti-atomism at best establish $\left(\mathrm{AA}_{2}\right)$ anti-atomism, as opposed $\left(\mathrm{AA}_{1}\right)$ anti-atomism.

Next consider 2), the inferential relationship between $\left(\mathrm{AA}_{2}\right)$ anti-atomism and D\&M-style instrumentalism/anti-realism about reference. It seems indisputable that $\left(\mathrm{AA}_{2}\right)$ anti-atomism, even permitting certain basic assumptions, does not entail (D\&M)style instrumentalism/anti-realism about reference. 
To be sure, $\left(\mathrm{AA}_{2}\right)$ anti-atomism, by way of the priority thesis, implies that the function of the concept of reference is in part to be a higher-level explanans and the corollary that reference is not the most fundamental or primitive concept in respect of explanatory significance (assuming such a linear ordering is possible). The problem is that $\left(\mathrm{AA}_{2}\right)$ anti-atomism does not entail that reference is merely a high-level explanans or a theoretical construct. To the extent that $\left(\mathrm{AA}_{2}\right)$ anti-atomism reigns supreme, i.e., at least some words are meaningful, it is far from obvious that the only purpose of the concept of reference is to characterize other semantic concepts, and in fact, it would seem considerably likely that the concept of reference serves both as an explanans and an explanandum.

In conciliation with this recognition, the concept of reference might plausibly be viewed similarly to other concepts that seemingly have a bilateral explanatory status, a status that seriously calls into question both instrumentalist and anti-realist readings of them. Some concepts in the physical sciences that designate unobservables appear to fall in this category: they have the dual explanatory status of serving both as higher-level explanans and lower-level explanandum. For example, consider atoms, electrons, protons, neutrons, etc. On the one hand, the concept of an atom, electron, etc. can be used to explain certain properties of macroscopic physical phenomena, while on the other hand, particular properties of atoms and electrons can be explained by more microscopic phenomena (e.g., quarks, superstrings, etc.). The point is that on the assumption of the bilateral explanatory status of atoms, electrons, etc., an assumption that is difficult to dispute, neither the instrumentalist reading of such concepts (according to which they are merely theoretical constructs) nor the anti-realist reading of them (according to which 
they do not designate existent objects) is compulsory. A virtually identical point applies to the concept of reference, mutatis mutandis.

In other words, the problem with the inference from $\left(\mathrm{AA}_{2}\right)$ anti-atomism to D\&M-style instrumentalism/anti-realism about reference is that it depends on a highly disputable theoretical assumption: reference, and possibly other concepts, cannot have a bilateral explanatory status - they cannot serve as both explanans and explanandum. It is worth noting, though, that so-called 'bilateralism' regarding the concept of reference seems both pre and post-reflectively tenable. On the post-reflective side, the appeal of the bilateral view of the concept of reference seems to stem from the fact that it is one part of an enticing comprehensive theoretical standpoint, what might be called 'holistic bilateralism' or 'local holism': the cognitive and explanatory significance of the semantic concepts of truth, reference, and meaning is in some way holistically constrained by their internal connections, i.e., it is not represented diagrammatically by an asymmetric, linear ordering, but by a type of multi-directional symmetry. ${ }^{16}$ Local holism, among other things, seems to have the virtue that it endorses a credible constraint on semantic theories, what Davies (1987) calls the 'mirror constraint', i.e., that any semantic theory must respect the structure of speaker understanding in the sense that if it is an empirical fact that our grasp of an expression $\mathrm{E}^{1}$ depends on our grasp of an expression $\mathrm{E}^{2}$, a semantic theory for our language has to assign meanings in such a way that the meaning of $\mathrm{E}^{1}$ depends on the meaning of $E^{2}$.

In sum, D\&M concede Field's premises that some form of physicalism is

\footnotetext{
${ }^{16}$ Such local holism should be kept distinct from the more radical thesis of global holism that is often alleged to be supported by Quinean arguments for the indeterminacy of reference/meaning: the thesis that the cognitive and explanatory significance of any expression e in a language $\mathrm{L}$ is holistically constrained by its connections with every other expression e in L.
} 
warranted, and that a physicalistically exemplary definition of truth should be physicalistically complete, but, on anti-atomism $\rightarrow$ referential instrumentalism/antirealism grounds, reject his conclusions that the semantic definition of truth is physicalistically and conceptually deficient. This reply to IC falls short since the antiatomism $\rightarrow$ referential instrumentalism/anti-realism thesis at its basis is far from unassailable. The problem with this thesis, it turns out, is that out of the two versions of anti-atomism, i) $\left(\mathrm{AA}_{1}\right)$ anti-atomism (which does entail referential instrumentalism/antirealism) is a contentious doctrine that seems a long way from being established by Quinean/Davidsonian inscrutability arguments, and ii) $\left(\mathrm{AA}_{2}\right)$ anti-atomism does not entail referential instrumentalism/anti-realism.

\section{§4 An Alternative Reply}

Our diagnosis of the failure of D\&M's reply to IC is suggestive of the kind of strategy required in replying to IC. D\&M's argument failed, in the first instance, because the antiatomism $\rightarrow$ referential instrumentalism/anti-realism inference at its basis effectively overlooked the relatively broad spectrum of appealing local holistic positions that surface between anti-atomism and an instrumentalism/anti-realism about reference. A minimally viable strategy must avoid this mistake and the tactical error it engenders - attempting to short-circuit the argument from Field's two premises to conclusion i). Assuming it would be injudicious to reject either of Field's two premises (a reasonable assumption in the current philosophical climate), the basic strategy required in replying to IC is less ambitious than that of D\&M: we should concede the two premises and conclusion i), but 
reject the inference from conclusion i) to conclusion ii). In other words, we should concede that physicalism is warranted (at least some variety of physicalism), that a physicalistically exemplary definition of truth should be physicalistically complete, and that the semantic definition of truth may be physicalistically incomplete, ${ }^{17}$ yet reject the conclusion that Tarski's definition is conceptually deficient. From the standpoint of a non-reductive-cum-local-holistic version of physicalism, the semantic definition of truth might well be viewed as both physicalistically incomplete and conceptual adequate.

The promise of this type of reply to IC begins to emerge upon recognition of the substructure of Field's argument. Field's argument from the two premises and conclusion i) to conclusion ii) is underwritten by a third implicit premise discussed above (in §2): definitions, at least of semantic concepts, are subject to a conceptual adequacy constraint — they must employ definiens more basic than their definiendum. As detailed, Field's argument does not get off the ground absent the assumption that the conceptual adequacy constraint governs, at a minimum, the definitions of semantic concepts. That neither Field nor any other proponent of IC that I am aware of has explicitly stated the conceptual adequacy constraint as a premise of IC, or supplied anything resembling a substantive defense of it, seems like a significant omission inasmuch as the assumption is far from incontrovertible.

In point of fact, the assumption at the basis of Field's argument - that the conceptual adequacy constraint governs either all definitions or definitions of semantic concepts - looks flawed on the face of it. The bottom line is that in the light of local

\footnotetext{
${ }^{17}$ I am leaving the question open of whether our local holistic position will dictate that the semantic definition of truth is physicalistically incomplete. The possibility cannot be ruled out, though, that by giving word meaning an explanatory role, local holism might reinstate Field's demand for a physicalistic
} 
holism, the appealing theoretical perspective both Field and D\&M failed to properly regard in arriving at their positions, the conceptual adequacy constraint on definitions can be dismissed outright.

To appreciate this, consider what the conceptual adequacy constraint amounts to. The conceptual adequacy constraint invokes what might be called an 'asymmetric' or 'bottom-up' requirement on definitions by way of its claim that definitions must employ definiens that are simpler or more primitive than the definiendum. In effect, the asymmetric requirement on definitions implies that any definition must be of the following reductive or explicit form: ' $\mathrm{X}$ ' means 'P', where 'P' must be a synonym of ' $\mathrm{X}$ ' that permits us to uniformly eliminate ' $\mathrm{X}$ ' from all contexts.

From the perspective of local holism, the problem with the conceptual adequacy constraint or any similar constraint on definitions is that it does not apply in full generality: its asymmetric-type requirement cannot be fulfilled for the concept of reference or any other semantic concept. Given local holist, internalist constraints, it is impossible to provide an explicit definition of 'refers to', one that replaces 'refers to' with more primitive synonyms that permit us to eliminate 'refers to' from all contexts in which it occurs. The crucial point is that defining the concept of reference in this way involves pinning it down externally, securing its explanatory foundations from outside the network of semantic concepts by way of an asymmetric, bottom-up reduction. Assuming, though, that the content of 'refers to' is holistically constrained by its position in the tripolar network of semantic concepts, its content is internal to the system, implying that reference cannot be defined by way of an asymmetric, explicit reduction (in

explanation of reference; from our perspective, such an explanation would need to be non-reductive, of course. 
a way that satisfies the conceptual adequacy constraint).

In short, assuming a local holistic framework, the conceptual adequacy constraint on definitions underlying Field's desiderata does not apply to any of the core semantic concepts, i.e., reference, meaning or truth. The implication is that (D) and (A), Tarski's definitions of reference (i.e., denotation and application), are neither in violation of any general conceptual adequacy constraint on definitions or particular desiderata on definitions of semantic concepts (at least that I am aware of). Contra Field, then, while (D) and (A) might be physicalistically incomplete, they do not appear to be conceptually deficient. Indeed, from our theoretical perspective, it turns out that (D) and (A) disclose the meaning of the concept of reference in the only way that is feasible: they furnish sets of axioms or principles involving the denotation and application predicates that fix their meaning at the object-level in an extensionally correct manner. Insofar as (D) and (A) are materially adequate (or extensionally correct), insofar as they correctly pair every name with its denotation and every predicate with its application conditions (the set of objects to which its applies), on local holism, they must be viewed as maximally conceptually illuminating, i.e., they capture the entire content of the concept of reference in the language(s) for it which it is being defined.

\section{$\$ 5$ Conclusion}

The best reply to IC involves conceding Field's premises that some form of physicalism is warranted (a non-reductive physicalism), and that a physicalistically exemplary definition of truth should be physicalistically complete, and conclusion i) that the 
semantic definition of truth is (or may be) physicalistically inadequate, but rejecting conclusion ii) that the semantic definition of truth is conceptually deficient. The argument from Field's premises and conclusion i) to conclusion ii) can be called into question inasmuch as it depends on an assumption that is inherently defective from the appealing perspective of local holism, the assumption that the conceptual adequacy constraint applies to the semantic concepts of truth, reference and meaning. On local holism, such an assumption can be dismissed out of hand; the implication being that Tarski's nonreductive, extensional definition of reference is conceptually sui generis.

Needless to say, the cogency of any such reply to IC is a direct function of the plausibility of local holism: if local holism flies wide of the mark, so does the reply. Local holism, it might also be stressed, is far from axiomatic: many will call into question the fundamental features of its semantic framework. In view of the pre and post-theoretic appeal of local holism, though, the proponent of IC is undoubtedly obligated to address our reply to IC by either taking aim at local holism or at the inference from local holism to the rejection of Field's fundamental desiderata, neither of which constitutes an obstacle easily surmountable. 


\section{References}

Benacerraf, P. (1973) 'Mathematical Truth'. Journal of Philosophy, 70, pp. 661-79.

Black, M. (1948) 'The Semantic Definition of Truth'. Analysis, 8, pp. 49-63.

Davidson, D. (1977) 'Reality without Reference'. Dialectica, 31, pp. 247-53. Reprinted in Inquiries into Truth and Interpretation (1984). Oxford: Oxford University Press. Page references are to the reprint.

- (1979) 'The Inscrutability of Reference'. Southwestern Journal of Philosophy, 10, pp. 7-19. Reprinted in Inquiries into Truth and Interpretation (1984). Oxford: Oxford University Press. Page references are to the reprint.

- (1996) 'The Folly of Trying to Define Truth'. Journal of Philosophy, 93(6).

Davies, M. (1987) Meaning, Quantification, and Necessity: Themes in Philosophical Logic. New York: Routledge and Kegan Paul.

Field, H. (1972) 'Tarski's Theory of Truth'. Journal of Philosophy, 69, pp. 347-75. - (1986) 'The Deflationary Conception of Truth'. Fact, Science, and Morality. ed. G. McDonald and C. Wright, Oxford: Basil Blackwell.

- (1994) 'Deflationist Views of Meaning and Content'. Mind, 103, pp. 249-85.

- (2001) Truth and the Absence of Fact. Oxford: Oxford University Press.

Harman, G. (1975) 'Meaning and Semantics'. Semantics and Philosophy. New York: New York University Press.

Leeds, S. (1978) 'Theories of Reference and Truth'. Erkenntnis, 13, pp. 111-29.

McDowell, J. (1978) 'Physicalism and Primitive Denotation: Field on Tarski'. Erkenntnis, 13, pp. 131-52.

Parsons, K. P. (1973) ‘Ambiguity and the Theory of Truth’. Nous, 7, pp. 379-94.

Putnam, H. (1975) 'The Meaning of Meaning'. Mind, Language, and Reality. Cambridge: Cambridge University Press.

- (1981) Reason, Truth, and History. Cambridge: Cambridge University Press.

Quine, W.V.O. (1969) Ontological Relativity and Other Essays. New York: Columbia University Press.

Soames, S. (1984) 'What is a Theory of Truth?' Journal of Philosophy, 81(8), pp. 411429.

Stalnaker, R. (1984) Inquiry. Cambridge, Massachusetts: MIT Press. 
Tarski, A. (1931) 'The Concept of Truth in Formalized Languages'. Reprinted in Logic, Semantics, Metamathematics: Papers from 1923 to 1928 (1983), Oxford: Oxford University Press, pp.152-278. Page references are to the reprint.

- (1944) 'The Semantic Conception of Truth and the Foundations of Semantics'. Philosophy and Phenomenological Research, 4, pp. 341-76. Reprinted in Logic, Semantics, Metamathematics: Papers from 1923 to 1928 (1983), Oxford: Oxford University Press, pp.152-278. Page references are to the original.

- (1983) Logic, Semantics, Metamathematics. 2nd ed. Indianapolis: Hackett.

Wolenski, J. (2001) 'In Defense of the Semantic Definition of Truth'. Synthese, 126, pp. 67-90. 